\title{
Small profinite m-stable groups
}

\author{
by
}

\section{Frank O. Wagner (Lyon)}

\begin{abstract}
A small profinite m-stable group has an open abelian subgroup of finite $\mathcal{M}$-rank and finite exponent.

1. Introduction. In a series of papers [7]-[12], Ludomir Newelski has developed the theory of multiplicity in analogy to the theory of independence. The basic set-up is that of a profinite structure (which may be thought of as a hyperdefinable set of algebraic hyperimaginaries), where he defines the notion of $m$-independence similarly to forking independence. This notion is automorphism invariant, symmetric, and transitive; if the ambient theory is small (with only countably many pure types), it also satisfies extension over finite sets. The corresponding foundation rank $\mathcal{M}$ has similar properties to Lascar rank in stability theory; a structure is m-stable (really, this should be m-superstable) if every type has ordinal $\mathcal{M}$-rank. Newelski asked two questions:
\end{abstract}

(1) $\mathcal{M}$-GAP CONJECTURE: In a small profinite structure, $\mathcal{M}(o)$ is either finite or $\infty$ for any orbit $o$.

(2) Does any small profinite group have an open abelian subgroup?

In this paper we shall prove the $\mathcal{M}$-gap conjecture for groups, and answer question (2) affirmatively in the m-stable case. In fact, we show:

THEOREM 1. A small m-stable profinite group has an open abelian subgroup, and is of finite $\mathcal{M}$-rank.

The line of argument follows the ideas in [4], where it is shown that a supersimple $\omega$-categorical group is finite-by-abelian-by-finite of finite $S U$ rank (which in turn was inspired by the $\omega$-stable case [1]). It also borrows some techniques of the bad group analysis from $[3,6,13]$.

2000 Mathematics Subject Classification: 20E18, 03C45.

Key words and phrases: profinite group, small theory, m-stability.

The author would like to thank Ludomir Newelski for helpful remarks. 
2. Profinite structures. We shall quickly review the basic definitions and properties we shall use. For a more detailed exposition, the reader may consult [11] or [12].

DeFinition 1. A profinite topological space is a compact Hausdorff topological space $U$ together with a system $\left(E_{i}: i<\omega\right)$ of refining equivalence relations with finitely many classes, such that:

- each $E_{i}$ is closed (as a subset of $U^{2}$ with the product topology),

- the $E_{i}$-classes form a basis of open sets for the topology.

(More generally, one should have a directed system of equivalence relations, but we shall restrict ourselves to the countable case.)

Let $\operatorname{Aut}_{0}^{*}(U)$ be the topological group of automorphisms of $U$ preserving all equivalence relations $\left(E_{i}: i<\omega\right)$, whose basic open subgroups are the stabilizers of finite subsets of $U$. A profinite structure is a pair $\left\langle U, \operatorname{Aut}^{*}(U)\right\rangle$, where $\operatorname{Aut}^{*}(U)$ is a closed subgroup of $\operatorname{Aut}_{0}^{*}(U)$; the group $\operatorname{Aut}^{*}(U)$ is called the structure group.

For a finite set $A$ of parameters, let $\operatorname{Aut}^{*}(U / A)$ be the group of automorphisms in $\operatorname{Aut}^{*}(U)$ fixing $A$ pointwise. A subset $X$ of $U$ is $A$-invariant if it is invariant under $\operatorname{Aut}^{*}(U / A)$; it is $A$-closed if it is closed and $A$-invariant. If $A=\emptyset$, it is usually omitted. A set is ${ }^{*}$-closed if it is $A$-closed for some finite $A$.

If $a$ is a finite tuple of elements of $U$, the orbit of $a$ under $\operatorname{Aut}^{*}(U / A)$ is denoted by $o(a / A)$.

Thus $A$-closed sets correspond to $A$-type-definable sets in ordinary model theory, and orbits correspond to types; moreover orbits are closed. Note that Newelski says $A$-definable instead of $A$-closed. As one really should say $A$ type-definable (the complement of a ${ }^{*}$-closed set need not be ${ }^{*}$-closed), we prefer our terminology.

Definition 2. A profinite structure is small if there are only countably many orbits on finite tuples over $\emptyset$.

Equivalently, we may ask that there are only countably many orbits on finite tuples, or just 1-orbits, over any finite set of parameters.

REMARK 2. In a small profinite structure, every $A$-closed set contains an open orbit over $A$.

Definition 3. The structure $U^{\text {eq }}$ is obtained from $U$ in the following way. For any $\emptyset$-closed equivalence relation on some $U^{n}$ we adjoin a new (imaginary) sort $U_{E}=U^{n} / E$, and a new function $\pi_{E}: U^{n} \rightarrow U_{E}$ mapping a tuple to its $E$-class. $U$ is identified with $U_{=}$. Then $\operatorname{Aut}^{*}(U)$ acts continuously on every sort, and hence on $U^{\text {eq }}$ (with the disjoint union topology). Every 
sort (with the induced structure group) is again a profinite structure, and $U^{\text {eq }}$ is a many-sorted profinite structure.

FACT 3 [11, Proposition 1.4]. Let $G$ be a group interpretable in a profinite structure $U^{\mathrm{eq}}$, i.e. its domain and the graphs of multiplication and inversion are $A$-closed for some finite $A$. Then $G$ is a profinite group, i.e. there are $A$-invariant open normal subgroups $G_{i}$ with $\bigcap_{i<\omega} G_{i}=\{1\}$ whose cosets form a basis of open sets for a compact Hausdorff topology.

ExAMPLE. Let $G$ be an $\omega$-saturated $\omega$-homogeneous group (possibly with additional structure), and $\left(G_{i}: i<\omega\right)$ a system of $\emptyset$-definable normal subgroups of finite index. Put $G^{0}=\bigcap_{i<\omega} G_{i}$. Then $G / G^{0}$ (with the induced structure group) is a profinite group; if $G$ is small, so is $G / G^{0}$. A subset of $G / G^{0}$ is $A$-closed iff it is induced by an $A$-type-definable subset of $G$.

From now on, $U$ will denote an infinite small profinite structure, and $G$ an infinite small profinite group. $A, B, \ldots$ will be finite sets of parameters, and $a, b, \ldots$ finite tuples (from $U^{\mathrm{eq}}$ or $G^{\mathrm{eq}}$, respectively).

FACT 4 [11, Lemma 2.2 and Proposition 2.3]. An A-invariant subgroup of $G$ is $A$-closed. The group generated by any family of A-invariant sets is A-closed, and generated in finitely many steps from finitely many sets. There is no infinite increasing chain of $A$-invariant subgroups of $G$. In particular all characteristic subgroups of $G$ are $\emptyset$-closed, and the ascending (upper) central series of $G$ becomes stationary after finitely many steps.

Corollary 5 [11, Proposition 2.4]. The intersection $G \cap \operatorname{acl}(A)$ is finite for all (finite) A. In particular, $G$ is locally finite.

Proof. $G \cap \operatorname{acl}(A)$ is an $A$-invariant subgroup, hence $A$-closed, and generated in finitely many steps from finitely many finite sets in $G \cap \operatorname{acl}(A)$.

Definition 4. A tuple $a \in U$ is $m$-independent of $B$ over $A$, denoted by $a^{m} \downarrow_{A} B$, if $o(a / A B)$ is open in $o(a / A)$. The $\mathcal{M}$-rank $\mathcal{M}$ is the least function from the collection of all orbits to the ordinals together with $\infty$ satisfying

$$
\mathcal{M}(a / A) \geq \alpha+1 \text { if there is } B \supseteq A \text { with } a \mathcal{L}_{A} B \text { and } \mathcal{M}(a / B) \geq \alpha .
$$

A theory is $m$-stable if every type has ordinal $\mathcal{M}$-rank.

FACT 6 ([11, Fact 1.10], [12, Lemma 1.5]). In a small profinite structure $U$,

(1) $m$-independence is symmetric and transitive,

(2) if $a \in \operatorname{acl}(A)$, then a $\mathfrak{L}_{A} B$ for all $B$,

(3) for any $a, A, B$ there is some $a^{\prime} \in o(a / A)$ with $a^{\prime} \downarrow_{A} B$,

(4) $\mathcal{M}(a / A, b)+\mathcal{M}(b / A) \leq \mathcal{M}(a, b / A) \leq \mathcal{M}(a / A, b) \oplus \mathcal{M}(b / A)$. 
Definition 5. Let $H$ be a ${ }^{*}$-closed subgroup of $G$. A *-closed subset $X$ of $H$ is generic (for $H$ ) if it is open in $H$. In particular, an orbit is generic (for $H$ ) if it is open in $H$.

Generic orbits exist by smallness (Remark 2); it is easy to see that if $o$ and $o^{\prime}$ are generic orbits for $H$, then $\mathcal{M}(o)=\mathcal{M}\left(o^{\prime}\right)$. We define $\mathcal{M}(H)=$ $\mathcal{M}(o)$, where $o$ is any generic orbit for $H$. In fact, the same reasoning works for coset spaces $G / H$, and $\mathcal{M}(G / H)=\mathcal{M}(o)$, where $o$ is any orbit open in $G / H$.

Remark 7. For two m-independent generic elements $g, h$ of $H$ the inverse $g^{-1}$ and the product $g h$ are both generic, and $g h$ is m-independent of $g$ and of $h$ (over any parameter set $A$ ).

Fact 6(4) immediately implies part (1) of Fact 8 below:

FACT 8 [11, Lemma 2.6]. Let $H$ be $a^{*}$-closed subgroup of $G$.

(1) $\mathcal{M}(H)+\mathcal{M}(G / H) \leq \mathcal{M}(G) \leq \mathcal{M}(H) \oplus \mathcal{M}(G / H)$.

(2) $H$ is open in $G$ iff $H$ has finite index in $G$.

(3) If $G$ is m-stable, then $H$ is open in $G$ iff $\mathcal{M}(H)=\mathcal{M}(G)$.

Hence if $G$ is m-stable, there is no infinite descending chain of *-closed subgroups, each of infinite index in its predecessor.

Here are two results whose proofs are more involved.

FACT 9 [11, Corollary 3.2]. If $G$ is m-stable, then $G$ has an infinite *closed abelian subgroup.

FACT 10 [11, Theorem 3.3]. If $G$ is m-stable and soluble, then $G$ has an open nilpotent subgroup.

Recall that two groups are commensurable if their intersection has finite index in either of them.

LEMma 11. Let $H_{a}$ be an a-closed subgroup of $G$, and suppose there is $a^{\prime} \in o(a)$ with $a^{\prime} m_{\downarrow}$ a such that $H_{a}$ and $H_{a^{\prime}}$ are commensurable. Let $E$ be the equivalence relation on o(a) given by $E\left(a^{\prime}, a^{\prime \prime}\right)$ if $H_{a^{\prime}}$ and $H_{a^{\prime \prime}}$ are commensurable. Then $E$ is closed, with finitely many classes, all of which are open; moreover, there is $n<\omega$ such that if $E\left(a^{\prime}, a^{\prime \prime}\right)$ holds, then $\left|H_{a^{\prime}}: H_{a^{\prime}} \cap H_{a^{\prime \prime}}\right| \leq n$.

Proof. Put $Y=o\left(a^{\prime} / a\right)$. By homogeneity, $o(a)$ is covered by $\emptyset$-conjugates of $Y$; by compactness finitely many conjugates suffice. This shows that $E$ has finitely many classes, which are all open, so $E$ is closed.

Moreover, if $a_{1}, a_{2} \in Y$, then the index of $H_{a_{i}} \cap H_{a}$ in $H_{a_{i}}$ and in $H_{a}$ equals the index of $H_{a^{\prime}} \cap H_{a}$ in $H_{a}$ and in $H_{a^{\prime}}$, for $i=1$, 2. It follows that the index of $H_{a_{1}} \cap H_{a_{2}}$ in $H_{a_{1}}$ and in $H_{a_{2}}$ is bounded independently of the 
choice of $a_{1}, a_{2}$. Since the same bound holds for all conjugates of $Y$, the lemma follows.

Note that the $E$-class $a_{E}$ of $a$ is a canonical parameter for the conjugacy class of $H_{a}$ in $G^{\text {eq }}$.

We finish this section with two purely group-theoretic theorems.

FACT 12 [5, Hauptsatz 7.6]. Let $G$ be a finite group, and $H$ a proper nontrivial subgroup such that $H \cap H^{g}=\{1\}$ for all $g \in G-H$. Then $N:=G-\bigcup_{g \in G}(H-\{1\})^{g}$ is a normal subgroup of $G$ with $G=N H$ and $N \cap H=\{1\}$.

FACT 13 [14, 2, 16, Theorem 4.2.4]. Let $G$ be any group, and $\mathfrak{H}$ a family of uniformly commensurable subgroups. Then there is a subgroup $N$ of $G, a$ finite extension of a finite intersection of groups in $\mathfrak{H}$ (and hence commensurable with them), such that $N$ is invariant under all automorphisms of $G$ fixing $\mathfrak{H}$ setwise.

3. Small profinite groups of finite $\mathcal{M}$-rank. Let $G$ be a profinite group.

Definition 6. A subgroup $H$ of $G$ is minimal if it is infinite, ${ }^{*}$-closed, and every ${ }^{*}$-closed subgroup of infinite index in $H$ is finite.

Note that in an m-stable profinite group every *-closed infinite subgroup of minimal $\mathcal{M}$-rank is minimal, so every ${ }^{*}$-closed subgroup contains a minimal one. By Fact 9 a minimal group has an open abelian subgroup.

Definition 7. Let $A$ and $B$ be abelian minimal subgroups of $G$. A virtual isogeny $f$ between $A$ and $B$ is a ${ }^{*}$-closed isomorphism $f: D / K \rightarrow I / C$, where $D$ is open in $A, I$ is open in $B$, and $K$ and $C$ are both finite. Two virtual isogenies $f_{1}$ and $f_{2}$ are equivalent, denoted by $f_{1} \sim f_{2}$, if the derived maps from $D_{1} \cap D_{2}$ to $\left(I_{1}+I_{2}\right) /\left(C_{1}+C_{2}\right)$ agree on an open subgroup of $A$.

Note that $f_{1}$ and $f_{2}$ are equivalent iff their graphs are commensurable. Equivalence of virtual isogenies is a congruence with respect to addition and composition (whenever composition makes sense). Moreover, an open subgroup, or a finite extension of a virtual isogeny (i.e. of the graph, as a subgroup of $A \times B$ ), is again a virtual isogeny, which is equivalent to the original one.

It is standard that in a minimal group $G$, the family of virtual autogenies (isogenies from $G$ to $G$ ) modulo equivalence, with addition and composition as operations, forms the set of invertible (nonzero) elements of a division ring $R$. (See [15] for this, and related results on virtual iso- and endogenies in small groups.) 
LEMMA 14. If $G$ is small, then $R$ is locally finite; for every a-closed virtual autogeny $f_{a}$ the equivalence relation $E(x, y)$ on o $(a)$ given by $f_{x} \sim f_{y}$ is * -closed and has finitely many classes, which are all open.

Proof. Let $\bar{f}$ be a finite tuple of virtual autogenies of $G$, and $\bar{a}$ a finite set of parameters over which $\bar{f}$ is defined. As $G$ is locally finite, we may replace every $f \in \bar{f}$ by a finite extension, and assume that it is defined on the whole of $G$ (we may have to increase $\bar{a}$ to do this). Choose $g \in G$ with $g{ }^{m} \bar{a}$. For any $f, f^{\prime} \in\langle\bar{f}\rangle$ we have $f(g), f^{\prime}(g) \in \operatorname{acl}(\bar{a}, g) \cap G$, which is finite. But if $f(g)=f^{\prime}(g)$, then $g \in \operatorname{ker}\left(f-f^{\prime}\right)$; as $g \notin \operatorname{acl}(\bar{a})$, the kernel of $f-f^{\prime}$ must be infinite, whence open by minimality, and $f \sim f^{\prime}$.

It follows that $R$ is locally finite, whence a (commutative) field. If $f_{a}$ is an $a$-closed virtual autogeny, then every $\emptyset$-conjugate of $f_{a}$ has the same order as $f_{a}$ modulo equivalence; as there are only finitely many elements in $R$ of that order, there must be $a^{\prime}{ }^{m} a$ with $f_{a} \sim f_{a^{\prime}}$. The rest follows from Lemma 11.

In particular, we can consider the equivalence class $\left(f_{a}\right)_{\sim}$ of a virtual autogeny as an imaginary element $a_{E}$.

THEOREM 15. Let $G$ be a small profinite abelian group of finite $\mathcal{M}$-rank. Then any *-closed subgroup of $G$ is commensurable with one invariant over some finite tuple in $\operatorname{acl}(\emptyset)$.

Proof. Consider first a minimal subgroup $A$ of $G$; say it is $a$-closed for some parameter $a$. By the finiteness of rank, there exist finitely many conjugates of $A$, say $\left(A_{i}: i<n\right)$, such that every conjugate of $A$ intersects $A^{0}:=\sum_{i<n} A_{i}$ in a subgroup of finite index. We may choose the $A_{i}$ almost linearly independent, i.e. $A_{i} \cap \sum_{j \neq i} A_{j}$ is finite for all $i<n$. Fix virtual isogenies $f_{i j}$ from $A_{i}$ to $A_{j}$ (whenever they exist), and let $\bar{a}$ be a finite set of parameters over which all of this is invariant.

Now consider another conjugate $A^{\prime}$ of $A$. Since $A^{\prime} \cap A^{0}$ is infinite by maximality of $n$, there must be some minimal $i=i\left(A^{\prime}\right)<n$ such that $A_{i} \cap\left(A^{\prime}+\sum_{k \neq i} A_{k}\right)$ is infinite; because $A$ and therefore $A_{i}$ are both minimal, $\left|A_{i}: A_{i} \cap\left(A^{\prime}+\sum_{k \neq i} A_{k}\right)\right|$ is finite. For every $j \neq i$ with $A_{j} \cap\left(A^{\prime}+\sum_{k \neq j} A_{k}\right)$ infinite we define a virtual isogeny $r\left(A^{\prime}, j\right)$ from $A_{i}$ to $A_{j}$ via: $r\left(A^{\prime}, j\right)(x)$ $:=\left\{y \in A_{j}: x-y \in A^{\prime}+\sum_{k \neq i, j} A_{k}\right\}=A_{j} \cap\left(x+A^{\prime}+\sum_{k \neq i, j} A_{k}\right)$ (it is easy to check from minimality that this is indeed a virtual isogeny). If $A_{j} \cap\left(A^{\prime}+\sum_{k \neq j} A_{k}\right)$ is finite, we put $r\left(A^{\prime}, j\right)=0$. Suppose now $A^{\prime \prime}$ is such that $i\left(A^{\prime \prime}\right)=i\left(A^{\prime}\right)$, and $r\left(A^{\prime}, j\right)$ and $r\left(A^{\prime \prime}, j\right)$ are equivalent virtual isogenies for all $j \neq i\left(A^{\prime}\right)$ with $r\left(A^{\prime}, j\right) \neq 0$ or $r\left(A^{\prime \prime}, j\right) \neq 0$. One can check that then $A^{\prime}$ and $A^{\prime \prime}$ must be commensurable.

By smallness we may choose $A^{\prime}$ such that $X:=o\left(a^{\prime} / \bar{a}\right)$ is open in $o(a)=o\left(a^{\prime}\right)$ (where the lower case letter denotes the parameter of the upper 
case group); note that if $a^{\prime \prime} \in X$, then $i\left(A^{\prime \prime}\right)=i\left(A^{\prime}\right)=: i$. Consider the equivalence relation $F_{j}\left(a^{\prime}, a^{\prime \prime}\right)$ on $X$ given by $f_{j i} \circ r\left(A^{\prime}, j\right) \sim f_{j i} \circ r\left(A^{\prime \prime}, j\right)$ for a fixed $j$. Since $f_{j i} \circ r\left(A^{\prime}, j\right)$ defines a virtual autogeny of $A_{i}$ for all $a^{\prime} \in X$, by Lemma 14 there are only finitely many $F_{j}$-classes. Hence there is $a^{\prime \prime} \in X$ with $a^{\prime \prime} \mathfrak{L}_{\bar{a}} a^{\prime}$ such that $F_{j}\left(a^{\prime}, a^{\prime \prime}\right)$ holds for all $j$, so $A^{\prime}$ and $A^{\prime \prime}$ are commensurable. But $a^{\prime} \underline{I}_{\perp} a^{\prime \prime}$; by Lemma 11 there are only finitely many commensurability classes among the $\emptyset$-conjugates of $A$, and each of them is uniformly commensurable.

By Fact 13 there is a ${ }^{*}$-closed subgroup $A^{c}$ commensurable with $A$ and invariant under all automorphisms of $G$ fixing the commensurability class of $A$. In other words, if $e \in \operatorname{acl}(\emptyset)$ is the canonical parameter for the conjugacy class of $A$, then $A^{c}$ is $e$-closed. This proves the assertion for minimal groups.

If $H \leq G$ is *-closed but not minimal, then by m-stability it contains a minimal subgroup $A$ which is commensurable with some acl $(\emptyset)$-definable $A^{c}$. But $H A^{c} / A^{c}$ is a subgroup of $G / A^{c}$ of smaller $\mathcal{M}$-rank; by induction it is commensurable with an $e^{\prime}$-closed group $H_{c} / A^{c}$, for some $e^{\prime} \in \operatorname{acl}(\emptyset)$, whose preimage $H^{c}$ in $G$ is as required.

LEMma 16. If $G$ is small and all centralizers of elements have finite index, then $G$ has an open abelian subgroup.

Proof. As $G \cap \operatorname{acl}(\emptyset)$ is finite, we may replace $G$ by an open subgroup and assume $G \cap \operatorname{acl}(\emptyset)=\{1\}$. For any $g \in G$, since $C_{G}(g)$ has finite index in $G$, we get $[g, G] \subseteq \operatorname{acl}(g)$. If $g^{m} \downarrow g^{\prime}$, then $\left[g, g^{\prime}\right] \in \operatorname{acl}(g) \cap \operatorname{acl}\left(g^{\prime}\right)=\operatorname{acl}(\emptyset)=\{1\}$. Since every element $g^{\prime}$ of $G$ can be written as $g^{\prime}=g_{1} g_{2}$ with $g^{m} g_{1}$ and $g^{m} \downarrow g_{2}$, we obtain $\left[g, g^{\prime}\right]=\left[g, g_{1} g_{2}\right]=\left[g, g_{2}\right]\left[g, g_{1}\right]^{g_{2}}=1$.

Proposition 17. A small profinite group of finite $\mathcal{M}$-rank has an open abelian subgroup.

Proof. Suppose not, and let $G$ be a counterexample of minimal $\mathcal{M}$-rank possible.

Claim. $G$ has an open soluble subgroup.

Proof of Claim. Suppose not. Note that if $H$ were an infinite ${ }^{*}$-closed subgroup of infinite index in $G$ with open normalizer, then $N_{G}(H) / H$ and $H$ would have open abelian normal subgroups by inductive hypothesis, and $G$ would have a 2-soluble open subgroup, a contradiction. Let $K$ be the subgroup of all elements $g$ whose centralizer $C_{G}(g)$ has finite index in $G$; this subgroup is $\emptyset$-invariant and hence closed by Fact 4 . Moreover, $K$ contains all finite subgroups whose normalizer is open in $G$. As $K$ is characteristic and cannot have finite index by Lemma 16, it is finite; after replacing $G$ by an open subgroup intersecting $K$ trivially, we may assume that $G$ has no nontrivial closed subgroup of infinite index whose normalizer is open in $G$. 
$G$ contains a minimal subgroup, and hence a ${ }^{*}$-closed abelian subgroup $B$, say, which we may take of maximal $\mathcal{M}$-rank possible; adding finitely many parameters, we assume $B$ is $\emptyset$-closed. Suppose $B^{\prime}$ is another *-closed abelian subgroup such that $B \cap B^{\prime}$ has infinite index in $B^{\prime}$. Now $C_{G}(b)$ is $b$-closed for any $b \in B \cap B^{\prime}$; since it contains $B$ and $B^{\prime}$, it has greater $\mathcal{M}$-rank than $B$ by the $\mathcal{M}$-rank inequalities. It therefore has no open abelian subgroup, and must be of finite index in $G$ by inductive hypothesis, whence $b=1$.

Let $N$ be the subgroup of all $g \in G$ such that $B^{g}$ is commensurable with $B$. It is $\emptyset$-invariant, and hence $\emptyset$-closed by Fact 4 ; note that the commensurability is uniform by Lemma 11: just consider $B^{g}$ and $B^{g^{\prime}}$ for generic m-independent $g, g^{\prime} \in N$. By Fact 13 there is a ${ }^{*}$-closed normal subgroup of $N$ commensurable with $B$, so $N$ cannot be open in $G$ by the first paragraph of the proof of the claim. Hence $N$ has an open abelian subgroup by inductive hypothesis, and $B$ is open in $N$ by maximality of $\mathcal{M}$-rank. It follows that there is an open $H \leq G$ such that $N \cap H \leq B$. Then $\mathcal{M}(B \cap H)=\mathcal{M}(B)$ and $B \cap H$ is commensurable with $(B \cap H)^{g}$ if and only if $B$ is commensurable with $B^{g}$, i.e. for $g \in N$. As $N \cap H=B \cap H$, we may thus replace $G$ by $H$ and assume that $B \cap B^{g}=\{1\}$ for any $g \in G-B$.

If $G_{0}$ is a finite subgroup of $G$ such that $B_{0}:=B \cap G_{0}$ is proper nontrivial, then $B_{0} \cap B_{0}^{g}=\{1\}$ for all $g \in G_{0}-B_{0}$. Suppose that there is a $G$-conjugate $B^{g}$ such that $B_{1}:=G_{0} \cap B^{g}$ is nontrivial, but not $G_{0^{-}}$-conjugate to $B_{0}$. As $B_{0}$ and $B_{1}$ are self-normalizing in $G_{0}$, and all $G_{0}$-conjugates of $B_{0}$ or $B_{1}$ intersect trivially, we get

$$
\begin{aligned}
\left|G_{0}\right| & \geq\left|G_{0} / B_{0}\right|\left(\left|B_{0}\right|-1\right)+\left|G_{0} / B_{1}\right|\left(\left|B_{1}\right|-1\right)+1 \\
& \geq 2\left|G_{0}\right|-\left|G_{0} / B_{0}\right|-\left|G_{0} / B_{1}\right|+1,
\end{aligned}
$$

whence $\left|G_{0} / B_{0}\right|+\left|G_{0} / B_{1}\right|>\left|G_{0}\right|$. We may assume $\left|B_{0}\right| \geq\left|B_{1}\right|$, and obtain

$$
\left|G_{0} / B_{1}\right| \geq\left|G_{0} / B_{0}\right|>\left|G_{0} / B_{1}\right|\left(\left|B_{1}\right|-1\right) \geq\left|G_{0} / B_{1}\right|,
$$

a contradiction. Hence all $B$-conjugates intersecting $G_{0}$ nontrivially are already conjugate in $G_{0}$.

Consider $X:=G-\bigcup_{g \in G}(B-\{1\})^{g}$. By the preceding paragraph, if $G_{0}$ is a finite subgroup of $G$ with $B_{0}:=G_{0} \cap B$ nontrivial, then $X \cap G_{0}=$ $G_{0}-\bigcup_{g \in G_{0}}\left(B_{0}-\{1\}\right)^{g}$; by Fact 12 this is a nontrivial normal subgroup of $G_{0}$. As $G$ is locally finite, $X$ is a nontrivial normal subgroup of $G$, which is invariant over the parameters used to define $B$, and thus ${ }^{*}$-closed by Fact 4 . Since it intersects $B$ trivially, it cannot be open, contradicting the conclusion of the first paragraph of the proof of the claim. This proves the assertion.

By Fact 10 we may assume that $G$ is nilpotent.

Claim. We may assume that $G^{\prime} \leq Z(G)$. 
Proof of Claim. By Fact 4 the subgroups $Z_{n}(G)$ in the upper central series are $\emptyset$-closed for all $n \geq 1$, and there is some minimal $n$ such that $Z_{n}(G)$ is infinite. Replacing $G$ by an open subgroup intersecting $Z_{n-1}(G)$ trivially, we may assume $n=1$. But now $\mathcal{M}(G / Z(G))<\mathcal{M}(G)$; by inductive hypothesis $G / Z(G)$ has an open abelian subgroup $H / Z(G)$ whose preimage $H$ in $G$ satisfies $H^{\prime} \leq Z(H)$.

For $g \in G$ put $H_{g}:=\{(h Z(G),[h, g]): h Z(G) \in G / Z(G)\}$, a subgroup of $G / Z(G) \times Z(G)$. Since $G / Z(G) \times Z(G)$ is abelian, $H_{g}$ is commensurable with an $e$-closed group for some $e \in \operatorname{acl}(\emptyset)$ by Theorem 15 . If $\pi_{1}$ denotes the projection onto the first coordinate, then $[h, g]=\left[h, g^{\prime}\right]$ for any $h Z(G) \in$ $\pi_{1}\left(H_{g} \cap H_{g^{\prime}}\right)$, whence $\left[h, g^{\prime} g^{-1}\right]=1$. However, we may choose $g$ and $g^{\prime}$ to be two independent generic elements such that $H_{g}$ and $H_{g^{\prime}}$ are commensurable. Then $\pi_{1}\left(H_{g} \cap H_{g^{\prime}}\right)$ is a subgroup of finite index in $G / Z(G)$, and $g^{\prime} g^{-1}$ is a generic element of $G$ with $\left|G: C_{G}\left(g^{\prime} g^{-1}\right)\right|$ finite.

The set of all $g \in G$ such that $C_{G}(g)$ has finite index in $G$ is a subgroup of $G$, which is $\emptyset$-invariant and closed; since it contains a generic element, it has finite index in $G$. Replacing $G$ by an open subgroup, we finish by Lemma 16.

Definition 8. A Morley sequence in an orbit $o$ over $A$ is a sequence $\left(a_{i}: i<\omega\right)$ of elements in the orbit such that $a_{i} \underline{I}_{A}\left(a_{j}: j<i\right)$ and $a_{k} \in o\left(a_{i} / A, a_{j}: j<i\right)$ for all $i \leq k<\omega$.

Note that if $o$ is over $A$, then in an m-stable theory there must be a finite $k<\omega$ such that $\mathcal{M}\left(A / a_{i}: i<k\right)$ is minimal possible. Then $A^{m} \downarrow_{\left(a_{i}: i<k\right)} a_{k}$; as $a_{k} \stackrel{m}{\perp}_{A}\left(a_{i}: i<k\right)$, the orbit $o\left(a_{k} / a_{i}: i<k\right)$ is parallel to $o$ (meaning that they have a common non-m-forking extension).

THEOREM 18. The $\mathcal{M - g a p}$ conjecture holds for small profinite groups: There is no orbit $o$ in a small profinite group with $\omega \leq \mathcal{M}(o)<\infty$. In particular, a small profinite $m$-stable group has finite $\mathcal{M}$-rank.

Proof. Let $G$ be a small profinite group containing a 1-orbit $o$ of infinite $\mathcal{M}$-rank $\alpha<\infty$. Taking $\mathrm{m}$-forking extensions if necessary, we may assume $\alpha=\omega$; adding parameters, we suppose that $o$ is over $\emptyset$. The subgroup of elements of finite $\mathcal{M}$-rank is $\emptyset$-invariant and hence closed by Fact 4; it follows that there is a bound $n<\omega$ on the $\mathcal{M}$-rank of a 1 -orbit over $\emptyset$ of finite $\mathcal{M}$-rank. Let $o^{\prime}$ be an $\mathrm{m}$-forking extension of $o$ of $\mathcal{M}$-rank $>n$, and $\left(a_{i}: i<\omega\right)$ a Morley sequence in $o^{\prime}$. Then there is $k<\omega$ such that $o\left(a_{k} / a_{i}: i<k\right)$ is parallel to $o^{\prime}$ and hence has $\mathcal{M}$-rank $>n$; it follows that there is a minimal $k<\omega$ such that over $\left(a_{i}: i \leq k\right)$ there is a 1-orbit of $\mathcal{M}$-rank $>n$. We add $\left(a_{i}: i<k\right)$ to the language. Then $n$ is the maximal $\mathcal{M}$-rank of a 1 -orbit of finite $\mathcal{M}$-rank over $\emptyset$, and there is $m>n$ which is 
the maximal $\mathcal{M}$-rank of a 1 -orbit of finite $\mathcal{M}$-rank over a single realization of $o\left(a_{k}\right)$ (which again we call $o$ ).

We repeat: Let $o^{\prime}$ be an m-forking extension of $o$ of $\mathcal{M}$-rank $>m$, and $\left(a_{i}: i<\omega\right)$ a Morley sequence in $o^{\prime}$. Let $\bar{a}=\left(a_{i}: i<k\right)$ be a maximal initial segment of $\left(a_{i}: i<\omega\right)$ which is m-independent over $\emptyset$. The groups $H\left(a_{i}\right)$ of elements of finite rank over $a_{i}$ are closed for all $i \geq k$, and conjugate under $\operatorname{Aut}^{*}(G / \bar{a})$. Let $H$ be the closed group of elements of finite rank over $\bar{a}, a_{k}$. Then $H\left(a_{i}\right) \leq H$ for $i \geq k$, so there are only finitely many commensurability classes for $H\left(a_{i}\right)$ with $i \geq k$ by Theorem 15 . Hence there are $i>j \geq k$ such that $H\left(a_{i}\right)$ and $H\left(a_{j}\right)$ are commensurable. But $\mathcal{M}\left(a_{i} / a_{j}\right) \geq \mathcal{M}\left(o^{\prime}\right)>m$, so $a_{i}{ }^{m} \downarrow a_{j}$ by the choice of $m$; by Lemma 11 and Fact 13 there is an imaginary $e \in \operatorname{acl}\left(a_{i}\right) \cap \operatorname{acl}\left(a_{j}\right)=\operatorname{acl}(\emptyset)$ and an $e$-closed subgroup $N$ commensurable with $H\left(a_{i}\right)$. But then for a generic element $g \in N$ we get $\mathcal{M}(g)=\mathcal{M}(g / e)=$ $\mathcal{M}(N)=\mathcal{M}\left(H\left(a_{i}\right)\right)=m>n$, a contradiction.

This concludes the proof of Theorem 1.

COROLlary 19. A small profinite m-stable group has finite exponent.

Proof. By Theorem 1, we may replace $G$ by an open subgroup and assume it is abelian. Let $o$ be an open orbit in $G$; by local finiteness its elements have finite order $n$, say. Then the group generated by $o$ is open in $G$ and has exponent $n$.

\section{References}

[1] W. Baur, G. Cherlin and A. Macintyre, Totally categorical groups and rings, J. Algebra 57 (1979), 407-440.

[2] G. M. Bergman and H. W. Lenstra, Jr., Subgroups close to normal subgroups, ibid. 127 (1989), 80-97.

[3] L.-J. Corredor, Bad groups of finite Morley rank, J. Symbolic Logic 54 (1989), 768-773.

[4] D. Evans and F. O. Wagner, Supersimple $\omega$-categorical groups and theories, ibid. 65 (2000), 767-776.

[5] B. Huppert, Endliche Gruppen, Springer, Berlin, 1967.

[6] A. Nesin, Non-soluble groups of Morley rank 3, J. Algebra 124 (1989), 199-218.

[7] L. Newelski, $\mathcal{M}$-rank and meager groups, Fund. Math. 150 (1996), 149-171.

[8] -, M-gap conjecture and m-normal theories, Israel J. Math. 106 (1998), 285-311.

[9] —, Geometry of * -finite types, J. Symbolic Logic 64 (1999), 1375-1395.

[10] —, m-normal theories, Fund. Math. 170 (2001), 141-163.

[11] —, Small profinite groups, J. Symbolic Logic 66 (2001), 859-872.

[12] —, Small profinite structures, Trans. Amer. Math. Soc. 354 (2002), 925-943.

[13] B. P. Poizat, Groupes Stables, Nur Al-Mantiq Wal-Ma'rifah, Villeurbanne, 1987.

[14] G. Schlichting, Operationen mit periodischen Stabilisatoren, Arch. Math. (Basel) 34 (1980), 97-99. 
[15] F. O. Wagner, Quasi-endomorphisms in small stable groups, J. Symbolic Logic 58 (1993), 1044-1051.

[16] —, Simple Theories, Math. Appl. 503, Kluwer, Dordrecht, 2000.

Institut Girard Desargues

Université Claude Bernard (Lyon-1)

Mathématiques

21 avenue Claude Bernard

69622 Villeurbanne Cedex, France

E-mail: wagner@desargues.univ-lyon1.fr

Received 22 August 2002;

in revised form 10 January 2003 